\title{
Occurrence and Distribution of Polybrominated Diphenyl Ethers in Sediments from Nairobi River Basin, Kenya, East Africa
}

Enock M. Osoro ${ }^{1 *}$, Shem O. Wandiga ${ }^{1}$, Vincent O. Madadi ${ }^{1}$, Deborah A. Abong' ${ }^{1}$

${ }^{1}$ College of Biological and Physical Sciences, School of Physical Science, Department of Chemistry, University of Nairobi, P.O. Box 30197-00100, Nairobi, Kenya

*Correspondence Author : osoroenock@yahoo.com

\section{ABSTRACT}

\section{Article Info}

Volume 8 Issue 1

Page Number: 274-286

Publication Issue :

January-February-2021

Article History

Accepted : 20 Feb 2021

Published : 28 Feb 2021
This study was set to determine the levels and spatial distribution of selected PBDEs in sediments of Nairobi River during the four seasons experienced in Nairobi. Sediment samples were collected from nine sites along the river and analyzed for brominated diphenyl ethers $28,47,66,85,99,100,153,154$, and 183 using gas chromatography coupled with mass spectrometer. The mean concentration of polybrominated diphenyl ethers residue in sediment samples ranged between $134.70 \pm 3.07$ to $24386.13 \pm 207.22 \mathrm{ng} / \mathrm{Kg}$. The high mean concentration of PBDEs in the river sediments indicates that anthropogenic activities along the Nairobi River basin have contributed to polybrominated diphenyl ethers contamination of the river sediment posing a potential risk to aquatic organisms that inhabit the river.

Keywords : Nairobi River Basin; Polybrominated diphenyl ethers; sediments; Residues

\section{INTRODUCTION}

Polybrominated diphenyl ethers (PBDEs) are used as flame retardants (FRs) in a range of manufactured goods like electronics and electrical goods, textiles, plastics, and furniture to decrease the risk of fire by interfering with the ignition of the polymeric materials [1,2]. PBDEs have 209 theoretically potential congeners in ten homologue groups (mono to deca) depending on the numbers and positions of the bromine atoms attaching on the two phenyl rings [3].There are three main commercial PBDE formulations namely pentabromodiphenyl ethers (Penta-BDE), octabromodiphenyl ethers (Octa-BDE), and decabromodiphenyl ethers (Deca-BDE) with the latter accounting for $83 \%$ of the total $\mathrm{PBDE}$ production internationally [3]. PBDEs are associated with adverse health effects that include thyroid hormone disruption [4], neuro-developmental deficit [5], liver problem [6], potential carcinogenesis [7], and abnormal pregnancy [8].

Recently there has been increased research on PBDEs because of the worldwide concern about their nondegradability, bioaccumulation, long-range transport, and toxicity in the atmosphere $[9,10]$. PBDEs contamination in the global environment was first detected in samples of fish caught in Swedish waters 
in 1981 [11]. Since then, various researchers have reported the existence of PBDEs in different ecological and living organism samples across the world. The research done includes; soil [12], sediments $[13,14]$ fish $[15,16]$, water $[17,18]$ wild aquatic species $[19,20]$ and mothers' milk [21]. Because of the non-degradability, bioaccumulation, toxicity, increased pollution levels of PBDEs in the environment, and risk to human health; penta, octa, and deca BDEs have been added in the Stockholm Convention on Persistent Organic Pollutants [22, 23]. Nairobi River is a river that flows through Kenya's capital city of Nairobi. It is the key river of the Nairobi river basin, a complex of numerous streams flowing eastwards. Nairobi River courses from Ondiri swamp on the Western part of Nairobi City and traverses settlements, the central business district, heavy and light industries in the mid and downstream and it eventually joins the Athi River at fourteen falls which drains to the Indian Ocean [24]. The river receives a conglomerate of wastes including; industrial waste released directly from factories at Nairobi's industrial area and light industries in Kariobangi [25], the garbage that includes unsorted waste electronics and electrical goods from Dandora dumping site, incorrectly treated sewages from Dandora sewage treatment plant [25], pesticides from urban agricultural activities [26], oil and grease, heavy metals, total petroleum hydrocarbons, polychlorinated biphenyl and other wastes from municipal and industrial activities [27, 28]. Nairobi River is the most contaminated river in Kenya [25].

There are few studies on environmental contamination by PBDEs in Kenya. Only few studies were found in the literature $[29,12]$. These studies focused on soil from suburban and rural areas of Kenya leaving the City of Nairobi where the contamination is expected to be high. Hence, research is needed to study the distribution of PBDEs in sediments from Nairobi River. The study will contribute to knowledge on the state of pollution of Nairobi River sediments with PBDEs and the largescale research findings can serve as a reference for relevant government agencies in policy formulation towards managing river resources.

The aim of this study was to determine the levels and spatial distribution of selected PBDEs in sediments of Nairobi River during the four seasons experienced in Nairobi.

\section{MATERIALS AND METHODS}

\subsection{Study Area}

Nairobi County is among the 47 counties in Kenya and is located between $36^{\circ} 45^{\prime \prime} \mathrm{E}$ to $37^{\circ} 05^{\prime} \mathrm{E}$, and $1^{\circ}$ $10^{\prime \prime S}$; to $1^{\circ} 30^{\prime \prime S}$ at a mean altitude of 1,700 meters above sea level. Commercial and administrative activities are concentrated in the central business district while most of the industrial activities are located in the South-East. Nairobi experiences four major seasons namely; hot dry season (January to March), heavy rain season (April to June), cool dry season (July to September), and short rain season (October to December). Temperatures generally vary from $11^{\circ} \mathrm{C}$ in June/July to about $29^{\circ} \mathrm{C}$ from December to March [30].

Nairobi River courses from Ondiri swamp on the Western part of Nairobi City and traverses the central business district, heavy and light industries, and settlements in the mid and downstream and it eventually joins the Athi River at fourteen falls which drains to the Indian Ocean. Figure 1 shows the distribution of the sampling locations along the river profile. 


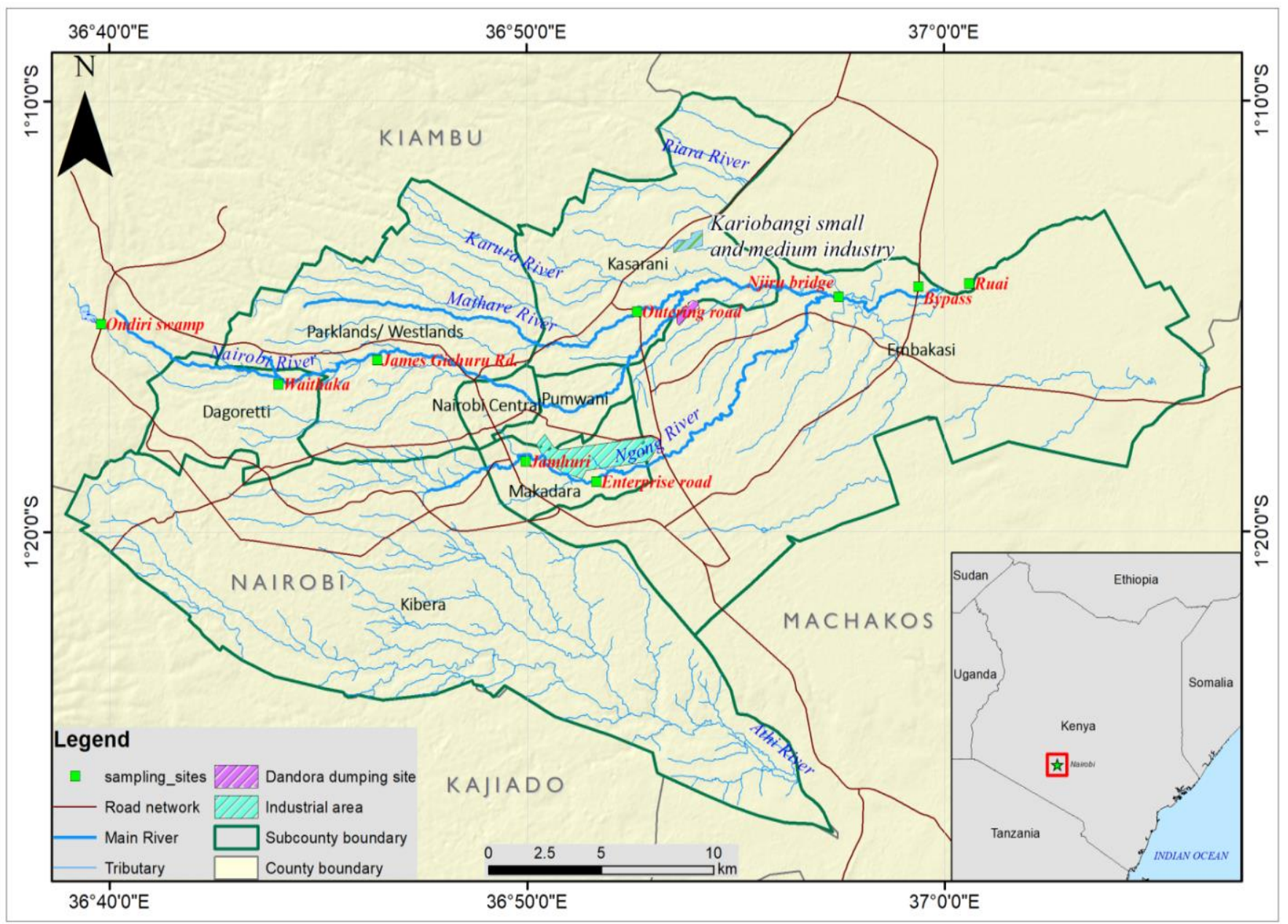

Figure 1 : Map of the study area showing the nine sampling sites along Nairobi River basin, i.e., Ondiri, Waithaka, James Gichuru, Jamhuri, Enterprise road, Outering road, Njiru, By-pass, and Ruai.

\subsection{Reagents and Chemicals}

Analytical grade silica gel (0.063-0.2 mesh, Merck), acetone, $\mathrm{n}$-hexane, sodium hydroxide, sulphuric acid, copper powder, anhydrous sodium sulphate, and dichloromethane were purchased from Sigma-Aldrich, USA through the local agent Kobian Kenya Ltd. HPLC grade isooctane was procured from SigmaAldrich, USA through the local agent Kobian Kenya Ltd. White sport nitrogen (99.999\%) gas and helium (99.999\%) gas were purchased from BOC Kenya Ltd. High purity (98.80\%) PBDEs standard mixture and ${ }^{13} \mathrm{C}$ labeled BDE 77 standard were procured from Sigma-Aldrich, USA. Sodium sulphate and neutral silica were purified by heating at $200{ }^{\circ} \mathrm{C}$ for 12 hours.

\subsection{Sample Collection}

Preliminary fieldwork was carried out before starting the sample collection to determine the exact sampling locations. Sampling locations were selected to cover the whole river profile and to represent the sediments of informal and formal settlement, industrial and commercial locations, dumping sites, and the source of the river. A total of nine sampling sites were selected to represent the upstream, midstream, and downstream of the river profile. Ondiri Swamp (0115'10S 036 39'48E), Waithaka (01 16'34S, 036응

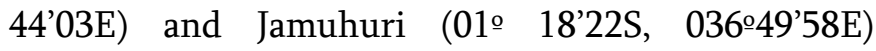
represented the upstream, James Gichuru Road (01응 18'36S, 036 49'42E), Enterprise road (01 18'22S, 036 49'35E) and Outering road (01 18'22S, 036 53'21E) represented the midstream while Njiru Bridge ( 01응 14'44S,036o 59'19E), By-Pass (01 14' 35S, 036o 56'27E) and Ruai (01 14'35S, 036 56'27E) represented the downstream (Figure 1). Sediments samples were collected quarterly from July 2017 to June 2018 covering the cool dry season (July-September), short 
rain season (October -December), hot dry season (January- March) and heavy rain season (April - June) to capture the four seasons experienced in Kenya [30]. Sediments were sampled using a pre-cleaned stainless steel shovel. Three composite samples were mixed on clean piece of aluminium foil and a $500 \mathrm{~g}$ representative sample picked, wrapped in another clean piece of aluminium foil, labelled, and then placed into a self-sealing bag. They were then packed in a cooling box and transported to the laboratory where they were stored in a deep freezer at $-20{ }^{\circ} \mathrm{C}$ prior to analysis [31]

\subsection{Extraction}

Sediment samples were removed from freezer and allowed to thaw for 4 hours prior to extraction. Triplicates of $20 \mathrm{~g}$ samples were dried with activated anhydrous sodium sulphate $\left(\mathrm{Na}_{2} \mathrm{SO}_{4}\right)$ overnight before transferring to the soxhlet thimble and then spiked with ${ }^{13} \mathrm{C}$ labelled BDE 77 extraction standard. This was extracted with $175 \mathrm{ml}$ of dichloromethane in a $200 \mathrm{ml}$ round bottomed flasks for 16 hours in a soxhlet extractor set-up. $2 \mathrm{ml}$ of isooctane added as keeper then concentrated to $3 \mathrm{ml}$ using LABCONCO rotary evaporator. The concentrated extracts were then put in vials and stored in a fridge at $-4{ }^{\circ} \mathrm{C}$ a waiting clean-up process.

\subsection{Sample Clean up}

Sample extracts were cleaned by passing them through a chromatographic glass column packed with $1 \mathrm{~cm}$ anhydrous sodium sulphate at the bottom, followed by $1000 \mathrm{mg}$ activated silica, $4 \mathrm{~g}$ basic silica (3:1 silica gel: 1M sodium hydroxide, weight/weight), $1000 \mathrm{mg}$ activated silica, $8 \mathrm{~g}$ acidic silica (1:1 silica gel: $\mathrm{H}_{2} \mathrm{SO}_{4}$, weight/weight), $2 \mathrm{~g}$ activated silica and on top $1 \mathrm{~cm}$ anhydrous $\mathrm{Na}_{2} \mathrm{SO}_{4}$. The column was preconditioned using $50 \mathrm{~mL}$ of $\mathrm{n}$-hexane. The extracts were quantitatively loaded and eluted with $50 \mathrm{~mL}$ of $\mathrm{n}$-hexane. $2 \mathrm{~mL}$ of isooctane was added to the cleaned samples and reduced to $2 \mathrm{~mL}$ then transferred to vial. Activated copper was added to remove the sulphur in the samples. The samples were then transferred into amber $1.5 \mathrm{~mL}$ autovials and further reduced under a gentle stream of nitrogen (99.99\%) to $0.5 \mathrm{~mL}$ for Gas Chromatography-Mass Spectrometry (GC-MS) analysis [31].

\subsection{Gas Chromatography-Mass Spectrometry (GC- MS) Analysis}

The samples were analyzed for 9 PBDE congeners (BDE 28, 47, 66, 99, 100, 85, 154, 153, and 183) using Agilent $6890 \mathrm{~N}$ gas chromatography (GC) equipped with a single quadrupole mass spectrometer (Agilent 5973A) and a Thermo scientific traceGOLD column (TG 5SILMS 30m X $0.25 \mathrm{~mm}$ X $0.25 \mu \mathrm{m}$ ). The mass spectrometer was operated in selected ion monitoring (SIM) mode with electron impact $\left(\mathrm{EI}^{+}\right)$ionization method at a resolution of $>5,000$. The detector and injection temperature was set at $320^{\circ} \mathrm{C}$ and $280^{\circ} \mathrm{C}$ in that order. High purity (99.999\%) helium N6 gas was used as the carrier gas at a flow rate of $1 \mathrm{ml} \mathrm{min}^{-1}$. The oven temperature was set at $90{ }^{\circ} \mathrm{C}(1$ minute hold time), then ramped from $90{ }^{\circ} \mathrm{C}$ to $180{ }^{\circ} \mathrm{C}$ at $40{ }^{\circ} \mathrm{C} \mathrm{min}$ ${ }^{1}$, ramped from $180{ }^{\circ} \mathrm{C}$ to $260{ }^{\circ} \mathrm{C}$ at $10{ }^{\circ} \mathrm{C} \min ^{-1}$ (2 minutes hold time), and finally ramped from $260{ }^{\circ} \mathrm{C}$ to $320{ }^{\circ} \mathrm{C}$ at $25{ }^{\circ} \mathrm{C} \mathrm{min}^{-1}$ (8 minutes hold time). 1 microliter of the sample extracts, mixed standard solutions, and calibration standards were injected into the Gas Chromatography-Mass Spectrometry (GC-MS) by splitless injection mode.

Identification of the targeted PBDE analytes was accomplished by relating the retention times and mass spectra of analytes in samples to those of reference standards analyzed at similar GC-MS conditions with the samples. The analytes were also identified using the National Institute of Standards and Technology (NIST) mass spectrometer library, version 2.0 (Standard reference data program of the US National Institute of Standards and Technology). A target PBDE was identified if it had a similar retention time to that of the reference standard (within a deviation of $\pm 0.05 \mathrm{~min}$ ) and their spectra matched. The peak identification was based on the 
base ions and the isotope pattern of each PBDE compound in the mass spectrometer spectra. The base ions were chosen as quantitative ions, while the other ions were selected as confirmatory ions (Table 1). Quantification of all target PBDE congeners was based on peak areas using external multilevel calibration curves prepared by plotting peak areas against the concentrations of the respective PBDE standard using nine calibration points with correlation coefficients $\left(\mathrm{r}^{2}\right)$ greater than 0.99 (Table 1).

Table 1 : Analytical figures of merit

\begin{tabular}{|c|c|c|c|c|c|c|c|c|c|}
\hline PBDEs & $\begin{array}{l}\text { BDE } \\
28\end{array}$ & $\begin{array}{l}\mathrm{BDE} \\
47\end{array}$ & $\begin{array}{l}\text { BDE } \\
66\end{array}$ & $\begin{array}{l}\text { BDE } \\
100\end{array}$ & $\begin{array}{l}\text { BDE } \\
99\end{array}$ & $\begin{array}{l}\text { BDE } \\
85\end{array}$ & $\begin{array}{l}\text { BDE } \\
154\end{array}$ & $\begin{array}{l}\text { BDE } \\
153\end{array}$ & $\begin{array}{l}\text { BDE } \\
183\end{array}$ \\
\hline $\begin{array}{l}\text { Retention } \\
\text { Time }\end{array}$ & 9.475 & 11.581 & 12.994 & 13.714 & 14.548 & 16.119 & 17.040 & 18.313 & 20.121 \\
\hline Linearity $\left(\mathrm{r}^{2}\right)$ & 0.998 & 0.997 & 0.990 & 0.996 & 0.998 & 0.994 & 0.992 & 0.998 & 0.997 \\
\hline LOD (pg) & 1.1 & 1.1 & 1.3 & 1.3 & 1.4 & 0.9 & 1.0 & 1.4 & 1.2 \\
\hline LOQ (pg) & 3.6 & 3.3 & 4.3 & 4.2 & 4.3 & 2.9 & 3.0 & 4.6 & 3.9 \\
\hline $\begin{array}{l}\text { Mean Ratio } \\
\text { of ion in } \\
\text { Sample/ } \\
\text { Std }\end{array}$ & 4.1 & 3.1 & 2.2 & 3.5 & 3.5 & 5.9 & 2.3 & 0.7 & 1.8 \\
\hline $\begin{array}{l}\text { Accuracy } \\
(\%)\end{array}$ & 98.14 & 102.86 & 105.06 & 101.51 & 97.89 & 89.35 & 104.31 & 103.58 & 102.23 \\
\hline $\begin{array}{l}\text { Quantitative } \\
\text { ion }(\mathrm{m} / \mathrm{z})\end{array}$ & 405 & 485.70 & 485.70 & 405.90 & 405.90 & 405.90 & 483.80 & 483.80 & 561.70 \\
\hline $\begin{array}{l}\text { Qualifier ion } \\
(\mathrm{m} / \mathrm{z})\end{array}$ & $\begin{array}{l}245.90 \\
407.80 \\
417.90 \\
419.90\end{array}$ & $\begin{array}{l}483.70 \\
6 \\
495.70 \\
6 \\
497.70 \\
345.90\end{array}$ & $\begin{array}{l}483.70, \\
495.70, \\
497.70 \\
345.90\end{array}$ & $\begin{array}{l}563.60, \\
565.60, \\
575.60, \\
577.60\end{array}$ & $\begin{array}{l}563.60 \\
565.60 \\
, \\
575.60 \\
577.60\end{array}$ & $\begin{array}{l}563.60, \\
565.60, \\
575.60, \\
577.60\end{array}$ & $\begin{array}{l}641.50, \\
643.50, \\
653.60 \\
655.50\end{array}$ & $\begin{array}{l}641.50, \\
643.50, \\
653.60, \\
655.50\end{array}$ & $\begin{array}{l}721.40, \\
733.40, \\
735.40, \\
723.40\end{array}$ \\
\hline
\end{tabular}

LOD, Limit of Detection

LOQ Limit of Quantification

\subsection{Quality assurance and quality control (QA/QC)}

All glassware and apparatus were washed using a detergent and water, and then rinsed with distilled water and solvents (methanol, acetone, and methylene chloride). The glassware were dried in the oven at $110^{\circ} \mathrm{C}$ for twelve hours before use. All reagents and solvents were of high purity and analytical grade. The PBDEs standard mixture and ${ }^{13} \mathrm{C}$ labelled BDE 77 standard were of high purity of above $98 \%$, kept in amber vials to prevent photo degradation, and stored in a freezer to prevent evaporation.

Many control tests were performed to authenticate the analytical procedures. Field blanks were carried to the field to track any contamination during transportation. The GC-MS machine was regularly injected with solvent blank (isooctane) and procedural blanks. A calibration standard check of 10 $\mathrm{ng} / \mathrm{L}$ was injected into the GC-MS after analysis of every ten samples to ensure that not more than fifteen per cent difference was established from the 
initial calibration standards. A recovery experiment was performed to test for the accuracy of the method. $1 \mathrm{ppm}{ }^{13} \mathrm{C}$ labelled BDE 77 standard was spiked to all samples before extraction and the average surrogate recovery in all samples was $89.96 \pm 11.32 \%$. The limit of detection was determined by reviewing the noise in the chromatograms next to the peak of interest. All PBDEs found with concentrations below the detection limit were reported as below detection limit (BDL). The limit of quantification (LOQ) was calculated in the same way using ten times the noise level (Table 1).

Microsoft Excel 2010 was used to process the data. Statistical Package for the Social Science (SPSS) version 20 for window evaluation was employed for the analysis of the data.

\section{RESULTS AND DISCUSSION}

Sediments were selected as matrices of interest for assessment since they acts as sinks for most hydrophobic organic contaminants, which strongly bind to the particulate matter owing to their high octanol-water partition coefficient (Kow) [32,33]. The analysis of sediment samples from nine sites along
Nairobi River showed presence of PBDEs residues at varying concentrations. The mean concentration of the $\sum 9 \mathrm{PBDEs}$ in sediment ranged between $134.70 \pm 3.07$ to $24386.13 \pm 207.22 \mathrm{ng} / \mathrm{Kg}$ (Table 2). PBDE levels measured in this study were higher compared to those reported by Olutona and Coworkers for Asunle Stream, Ile-Ife, Nigeria, where the mean concentration of the $\sum_{6} \mathrm{PBDEs}$ in the sediments ranged between 0.83 to $10.45 \mathrm{ng} / \mathrm{g}$ [34] and surface sediment samples from a recharge point of Guarani Aquifer in Ribeirão Preto, Brazil whose concentration ranged from nd $-5.4 \mathrm{ng} / \mathrm{g}$ [35]. However, the levels in the current study were lower than those reported in surface sediment samples from from Lake Chaohu, central eastern China whose concentration ranged from 8.93-45 ng/g [36], sediment from the artificial Lake Shihwa, Korea whose concentration ranged from 1.13-18700 $\mathrm{ng} / \mathrm{g}$ [37], those in the river and coastal areas of Portugal whose concentration ranged from 185.50 to $277.20 \mathrm{mg} / \mathrm{kg}$ [37], Osaka Bay of Japan whose concentration ranged from 8.80 to $13.52 \mathrm{mg} / \mathrm{kg}$ [38] and those in San Francisco Estuary of US whose concentration ranged from ND to $2.12 \mathrm{mg} / \mathrm{kg}$ [39]. The mean concentrations of PBDEs in this study were below Environment Canada's (EC's) safe limit (6124 $\mathrm{ng} / \mathrm{g}$ ) [40].

Table 2 : Range and Mean Concentration of PBDEs Residue Level in Sediments (ng/Kg)

\begin{tabular}{|c|c|c|c|c|c|c|c|c|c|c|}
\hline $\begin{array}{r}\text { Site/ } \\
\text { PBDE }\end{array}$ & & BDE 28 & $\begin{array}{r}\text { BDE } \\
47 \\
\end{array}$ & $\begin{array}{r}\mathrm{BDE} \\
66 \\
\end{array}$ & $\begin{array}{r}\text { BDE } \\
100 \\
\end{array}$ & BDE 99 & $\begin{array}{r}\mathrm{BDE} \\
85 \\
\end{array}$ & $\begin{array}{r}\mathrm{BDE} \\
154 \\
\end{array}$ & BDE 153 & $\begin{array}{r}\text { BDE } \\
183 \\
\end{array}$ \\
\hline \multirow{2}{*}{ Ondiri } & Range & $\begin{array}{l}216.27- \\
1321.44\end{array}$ & $\begin{array}{l}1051.23- \\
6521.00\end{array}$ & $\begin{array}{l}191.55- \\
718.43\end{array}$ & $\begin{array}{l}478.48- \\
1232.15\end{array}$ & $\begin{array}{l}1021.53- \\
3059.17\end{array}$ & $\begin{array}{l}134.70- \\
538.22\end{array}$ & $\begin{array}{l}352.63- \\
2813\end{array}$ & $\begin{array}{l}233.48- \\
1645.22\end{array}$ & $\begin{array}{l}653.87- \\
864.27\end{array}$ \\
\hline & $\begin{array}{r}\text { Mean } \pm \\
\text { SD }\end{array}$ & $\begin{array}{l}860.79 \\
\pm 463.60\end{array}$ & $\begin{array}{l}3871.46 \pm \\
2968.49\end{array}$ & $\begin{array}{l}515.70 \pm \\
235.11\end{array}$ & $\begin{array}{l}824.56 \pm \\
363.28\end{array}$ & $\begin{array}{l}12505.76 \\
\pm 1349.87\end{array}$ & $\begin{array}{l}296.96 \pm \\
192.84\end{array}$ & $\begin{array}{l}1078.65 \\
\pm 1161.9\end{array}$ & $\begin{array}{l}701.65 \pm 6 \\
54.02\end{array}$ & $\begin{array}{l}727.04 \pm \\
93.52\end{array}$ \\
\hline \multirow{2}{*}{ Jamhuri } & Range & $\begin{array}{l}791.75- \\
1688.62\end{array}$ & $\begin{array}{l}1116.73- \\
8733.92\end{array}$ & $\begin{array}{l}464.78- \\
1785.67\end{array}$ & $\begin{array}{l}758.45- \\
1687.27\end{array}$ & $\begin{array}{l}876.89- \\
5742.18\end{array}$ & $\begin{array}{l}582.51- \\
1758.99\end{array}$ & $\begin{array}{l}987.72- \\
3699.28\end{array}$ & $\begin{array}{l}681.06- \\
3913.25\end{array}$ & $\begin{array}{l}478.38- \\
1536.18\end{array}$ \\
\hline & $\begin{array}{r}\text { Mean } \pm \\
\text { SD }\end{array}$ & $\begin{array}{l}1232.09 \\
\pm 366.32\end{array}$ & $\begin{array}{l}5035.78 \\
\pm 3894.92\end{array}$ & $\begin{array}{l}1097.08 \\
\pm 625.05\end{array}$ & $\begin{array}{l}1236.87 \\
\pm 395.87\end{array}$ & $\begin{array}{l}3437.43 \pm \\
2260.99\end{array}$ & $\begin{array}{l}911.04 \pm \\
566.26\end{array}$ & $\begin{array}{l}2182.61 \\
\pm 1294.7\end{array}$ & $\begin{array}{l}1775.11 \pm \\
1491.99\end{array}$ & $\begin{array}{l}940.22 \pm \\
498.83\end{array}$ \\
\hline \multirow{2}{*}{ Waithaka } & Range & $\begin{array}{l}524.36- \\
1532.4\end{array}$ & $\begin{array}{l}1517.75- \\
6980.11\end{array}$ & $\begin{array}{l}141.67- \\
1294.00\end{array}$ & $\begin{array}{l}330.64- \\
711.51\end{array}$ & $\begin{array}{l}1087.44- \\
5196.00\end{array}$ & $\begin{array}{l}364.68- \\
924.20\end{array}$ & $\begin{array}{l}738.19- \\
1247.23\end{array}$ & $\begin{array}{l}371.64- \\
1725.64\end{array}$ & $\begin{array}{l}761.89- \\
2315.00\end{array}$ \\
\hline & $\begin{array}{r}\text { Mean } \pm \\
\text { SD }\end{array}$ & $\begin{array}{r}1018.78 \\
\pm 433.26\end{array}$ & $\begin{array}{l}4041.02 \\
\pm 2932.24\end{array}$ & $\begin{array}{l}662.49 \pm \\
475.63\end{array}$ & $\begin{array}{l}445.54 \pm \\
206.65\end{array}$ & $\begin{array}{r}13050.73 \\
\pm 2239.57\end{array}$ & $\begin{array}{l}661.61 \pm \\
299.55\end{array}$ & $\begin{array}{l}1161.93 \\
\pm 379.12\end{array}$ & $\begin{array}{l}893.67 \pm 5 \\
83.06\end{array}$ & $\begin{array}{l}1187.58 \\
\pm 753.52\end{array}$ \\
\hline \multirow{2}{*}{$\begin{array}{r}\text { James } \\
\text { Gichuru } \\
\text { Road }\end{array}$} & Range & $\begin{array}{l}515.67- \\
1165.42 \\
\end{array}$ & $\begin{array}{l}1792.16- \\
8912.09\end{array}$ & $\begin{array}{l}281.76- \\
1826.03 \\
\end{array}$ & $\begin{array}{l}1526.29 \\
-1843.1 \\
\end{array}$ & $\begin{array}{l}1839.76- \\
10785.00\end{array}$ & $\begin{array}{l}361.00- \\
1224.11\end{array}$ & $\begin{array}{l}816.91- \\
2526.00\end{array}$ & $\begin{array}{l}586.70- \\
2226.21\end{array}$ & $\begin{array}{l}271.54- \\
1981.99\end{array}$ \\
\hline & $\begin{array}{r}\text { Mean } \pm \\
\text { SD }\end{array}$ & $\begin{array}{r}1214.30 \\
\pm 830.65\end{array}$ & $\begin{array}{l}5684.26 \\
\pm 3272.53\end{array}$ & $\begin{array}{l}879.58 \pm \\
666.10\end{array}$ & $\begin{array}{l}1771.86 \\
\pm 300.41\end{array}$ & $\begin{array}{l}4960.32 \pm \\
3973.66\end{array}$ & $\begin{array}{l}805.44 \pm \\
428.50\end{array}$ & $\begin{array}{l}1269.08 \\
\pm 838.28\end{array}$ & $\begin{array}{l}1135.62 \pm \\
747.96\end{array}$ & $\begin{array}{l}939.26 \pm \\
828.45\end{array}$ \\
\hline
\end{tabular}




\begin{tabular}{|c|c|c|c|c|c|c|c|c|c|c|}
\hline \multirow{2}{*}{$\begin{array}{r}\text { Enterprise } \\
\text { Road }\end{array}$} & Range & $\begin{array}{l}1683.65 \\
-2382.6 \\
\end{array}$ & $\begin{array}{l}10772.95 \\
-12332.5 \\
\end{array}$ & $\begin{array}{l}681.91- \\
2556.37\end{array}$ & $\begin{array}{l}\text { 681.91- } \\
2556.37 \\
\end{array}$ & $\begin{array}{l}4843.61- \\
10082.93 \\
\end{array}$ & $\begin{array}{l}1168.27 \\
-2867.6 \\
\end{array}$ & $\begin{array}{l}1564.46 \\
-5736.1 \\
\end{array}$ & $\begin{array}{l}1257.73- \\
3687.57 \\
\end{array}$ & $\begin{array}{l}1156.76 \\
-2167.1 \\
\end{array}$ \\
\hline & $\begin{array}{r}\text { Mean } \pm \\
\text { SD }\end{array}$ & $\begin{array}{l}1978.01 \\
\pm 302.93\end{array}$ & $\begin{array}{l}11608.75 \\
\pm 751.06\end{array}$ & $\begin{array}{l}1622.63 \\
\pm 911.44 \\
\end{array}$ & $\begin{array}{l}1622.63 \\
\pm 911.44 \\
\end{array}$ & $\begin{array}{l}8260.61 \pm \\
2364.73\end{array}$ & $\begin{array}{l}1738.36 \\
\pm 776.90 \\
\end{array}$ & $\begin{array}{l}2314.03 \\
\pm 2357.6 \\
\end{array}$ & $\begin{array}{l}2332.64 \pm \\
1006.51\end{array}$ & $\begin{array}{l}1715.84 \\
\pm 417.19\end{array}$ \\
\hline \multirow{2}{*}{$\begin{array}{r}\text { Outering } \\
\text { Road }\end{array}$} & Range & $\begin{array}{l}1262.81 \\
-1886.7\end{array}$ & $\begin{array}{l}6692.03- \\
10483.16\end{array}$ & $\begin{array}{l}682.69- \\
2476.83\end{array}$ & $\begin{array}{l}967.00- \\
1362.15\end{array}$ & $\begin{array}{l}716.94- \\
11914.72 \\
\end{array}$ & $\begin{array}{l}1119.56 \\
-1716.2\end{array}$ & $\begin{array}{l}1700.31 \\
-3916.3\end{array}$ & $\begin{array}{l}1172.34- \\
3916.30\end{array}$ & $\begin{array}{l}1054.80 \\
-3279.9\end{array}$ \\
\hline & $\begin{array}{r}\text { Mean } \pm \\
\text { SD }\end{array}$ & $\begin{array}{l}1982.75 \\
\pm 936.45\end{array}$ & $\begin{array}{l}9593.26 \\
\pm 2163.92\end{array}$ & $\begin{array}{l}1709.68 \\
\pm 757.85\end{array}$ & $\begin{array}{l}12737.0 \\
7 \pm 4174\end{array}$ & $\begin{array}{l}10051.19 \\
\pm 1795.85\end{array}$ & $\begin{array}{l}1400.51 \\
\pm 245.58\end{array}$ & $\begin{array}{l}2533.43 \\
\pm 1005.4\end{array}$ & $\begin{array}{l}1991.31 \pm \\
1300.43\end{array}$ & $\begin{array}{l}1828.03 \\
\pm 1022.7\end{array}$ \\
\hline \multirow{2}{*}{ By-Pass } & Range & $\begin{array}{l}1954.36 \\
-3816.8 \\
\end{array}$ & $\begin{array}{l}8968.24- \\
14117.73\end{array}$ & $\begin{array}{l}1669.87 \\
-3226.2\end{array}$ & $\begin{array}{l}1262.23 \\
-3491.3\end{array}$ & $\begin{array}{l}5731.03- \\
12856.34\end{array}$ & $\begin{array}{l}952.41- \\
2554.23\end{array}$ & $\begin{array}{l}1328.61 \\
-3851.7\end{array}$ & $\begin{array}{l}1841.91- \\
4213.69\end{array}$ & $\begin{array}{l}1384.45 \\
-2616.3\end{array}$ \\
\hline & $\begin{array}{r}\text { Mean } \pm \\
\text { SD } \\
\end{array}$ & $\begin{array}{l}2515.14 \\
\pm 877.88 \\
\end{array}$ & $\begin{array}{l}10656.23 \\
\pm 2366.47 \\
\end{array}$ & $\begin{array}{l}2260.27 \\
\pm 691.45\end{array}$ & $\begin{array}{l}1900.57 \\
\pm 1068.3\end{array}$ & $\begin{array}{l}9468.08 \pm \\
2975.65 \\
\end{array}$ & $\begin{array}{l}1687.33 \\
\pm 658.98 \\
\end{array}$ & $\begin{array}{l}2420.17 \\
\pm 1094.6 \\
\end{array}$ & $\begin{array}{l}3324.55 \pm \\
1043.42 \\
\end{array}$ & $\begin{array}{l}2016.93 \\
\pm 648.41 \\
\end{array}$ \\
\hline \multirow{2}{*}{ Njiru } & Range & $\begin{array}{l}1345.30 \\
-3362.4 \\
\end{array}$ & $\begin{array}{l}8955.9- \\
24386.1\end{array}$ & $\begin{array}{l}1768.59 \\
-3971.4 \\
\end{array}$ & $\begin{array}{l}1731.89 \\
-2832.0 \\
\end{array}$ & $\begin{array}{l}6961.86- \\
11245.21 \\
\end{array}$ & $\begin{array}{l}1296.74 \\
-2719.5 \\
\end{array}$ & $\begin{array}{l}2816.99 \\
-4752.2 \\
\end{array}$ & $\begin{array}{l}1861.51- \\
5497.66\end{array}$ & $\begin{array}{l}1520.14 \\
-3116.9 \\
\end{array}$ \\
\hline & $\begin{array}{r}\text { Mean } \pm \\
\text { SD } \\
\end{array}$ & $\begin{array}{l}2475.48 \\
\pm 853.71 \\
\end{array}$ & $\begin{array}{l}13686.91 \\
\pm 7189.32 \\
\end{array}$ & $\begin{array}{l}2635.09 \\
\pm 1018.5 \\
\end{array}$ & $\begin{array}{l}2107.50 \\
\pm 556.08 \\
\end{array}$ & $\begin{array}{l}9380.82 \pm \\
1777.98 \\
\end{array}$ & $\begin{array}{l}1884.55 \\
\pm 607.69 \\
\end{array}$ & $\begin{array}{l}3665.49 \\
\pm 810.87 \\
\end{array}$ & $\begin{array}{l}3224.94 \pm \\
1620.95 \\
\end{array}$ & $\begin{array}{l}12029.7 \\
0 \pm 743.2\end{array}$ \\
\hline \multirow{2}{*}{ Ruai } & Range & $\begin{array}{l}1731.88 \\
-3095.2\end{array}$ & $\begin{array}{l}6874.90- \\
10556.99 \\
\end{array}$ & $\begin{array}{l}1119.79 \\
-3331.6 \\
\end{array}$ & $\begin{array}{l}1334.52 \\
-2786.1\end{array}$ & $\begin{array}{l}8445.89- \\
10668.32 \\
\end{array}$ & $\begin{array}{l}1015.74 \\
-2554.3\end{array}$ & $\begin{array}{l}2154.19 \\
-3914.2\end{array}$ & $\begin{array}{l}1519.88- \\
3648.79\end{array}$ & $\begin{array}{l}1199.74 \\
-3007.7\end{array}$ \\
\hline & $\begin{array}{r}\text { Mean } \pm \\
\text { SD } \\
\end{array}$ & $\begin{array}{l}2309.06 \\
\pm 571.07\end{array}$ & $\begin{array}{l}9248.14 \\
\pm 1676.06 \\
\end{array}$ & $\begin{array}{l}2372.47 \\
\pm 1072.1 \\
\end{array}$ & $\begin{array}{l}12089.1 \\
4 \pm 620.9\end{array}$ & $\begin{array}{l}9651.81 \pm \\
1066.34 \\
\end{array}$ & $\begin{array}{l}1726.40 \\
\pm 642.92\end{array}$ & $\begin{array}{l}2780.32 \\
\pm 810.20\end{array}$ & $\begin{array}{l}2635.29 \pm \\
909.44\end{array}$ & $\begin{array}{l}2509.26 \\
\pm 874.55 \\
\end{array}$ \\
\hline $\begin{array}{l}\text { Detection } \\
\text { Frequency }\end{array}$ & $\%$ & 100 & 100 & 100 & 100 & 100 & 100 & 100 & 100 & 100 \\
\hline
\end{tabular}

$\mathrm{SD}$, standard deviation; BDL, below detection limit, $\mathrm{n}=3$

\subsection{Spatial and Seasonal Variation of PBDEs Residue Level in Sediment}

The mean concentrations of PBDEs in sediment at the nine sampling sites along Nairobi river basin were assessed to measure their variation designs. The data gotten for the nine sampling sites are shown in Table 3 . The result showed that the PBDE congeners were portable and can be moved over a long distance over period. Additionally, the nine PBDEs investigated were present in all of the nine sampling sites in the river at different concentrations. Spatially, the average concentration of the total PBDEs ranged between 1191.57 and $4400.49 \mathrm{ng} / \mathrm{Kg}$.

Njiru site situated downstream of the river recorded the highest level of PBDEs among the nine locations which was attributed to leaking of the PBDEs from open burning of wastes including plastics at Dandora dumpsite and discharge of industrial wastewater from light industries at Kariobangi.

Generally, from the source of the river (Ondiri), the mean concentration of these contaminants increased downstream. The sampling sites; Ruai, By-pass, Njiru, Outering road and Enterprise road had relatively higher concentration of PBDEs that is associated with their close proximity to Dandora municipal dumpsite where unsorted wastes are dumped including plastics, domestic and industrial wastes hence leaching of the contaminants into the receiving river is highly probable. The upstream section of Nairobi River had relatively low concentration of PBDEs that is attributed to atmospheric deposition from open burning of solid wastes. This suggests that atmospheric deposition is a route of movement of particulate pollutants to Nairobi River's ecosystem [41]. Also these could be attributed to the varying distances of the sampling locations to the source of contaminants. 
Table 3 : Mean Levels (ng/Kg) of the Spatial Allocation of PBDEs in the Nairobi River Sediment Samples

\begin{tabular}{|l|l|l|l|l|l|l|l|l|l|l|}
\hline & & $\mathrm{BDE}$ & $\mathrm{BDE}$ & $\mathrm{BDE}$ & $\mathrm{BDE}$ & $\mathrm{BDE}$ & $\mathrm{BDE}$ & $\mathrm{BDE}$ & $\mathrm{BDE}$ & $\begin{array}{l}\text { 9 } \\
\end{array}$ \\
& $\mathrm{BDE} 28$ & 47 & 66 & 85 & 99 & 100 & 153 & 154 & 183 & PBDEs \\
\hline & 860.78 & 3871.46 & 515.69 & 296.93 & 2505.75 & 824.56 & 701.65 & 1078.65 & 68.62 & \\
Ondiri & \pm 463.60 & \pm 2968.49 & \pm 235.12 & \pm 192.84 & \pm 1349.87 & \pm 363.28 & \pm 654.02 & \pm 1161.93 & \pm 93.52 & 1191.57 \\
\hline & 1018.78 & 4041.02 & 662.48 & 661.60 & 3050.73 & 445.54 & 893.67 & 1161.93 & 563.71 & \\
Waithaka & \pm 433.26 & \pm 2932.24 & \pm 475.63 & \pm 299.54 & \pm 2239.56 & \pm 206.65 & \pm 583.06 & \pm 379.12 & \pm 753.52 & 1388.83 \\
\hline James & 1214.29 & 5684.26 & 879.58 & 805.44 & 4960.31 & 1771.87 & 1135.62 & 1269.08 & 666.24 & \\
Gichuru & \pm 830.64 & \pm 3272.52 & \pm 666.11 & \pm 428.50 & \pm 3973.66 & \pm 300.41 & \pm 747.96 & \pm 838.28 & \pm 828.45 & 2042.97 \\
\hline & 1232.09 & 5035.77 & 1097.08 & 911.04 & 3437.42 & 1236.86 & 1775.11 & 2182.80 & 409.81 & \\
Jamhuri & \pm 366.32 & \pm 3894.92 & \pm 625.05 & \pm 566.26 & \pm 2260.99 & \pm 395.56 & \pm 1491.99 & \pm 1294.78 & \pm 468.83 & 1924.22 \\
\hline & 1982.74 & 9593.26 & 1709.68 & 2900.51 & 10051.18 & 1187.07 & 1991.31 & 2533.43 & 725.95 & \\
Outering & \pm 936.74 & \pm 2163.92 & \pm 757.85 & \pm 3017.9 & \pm 1795.85 & \pm 215.69 & \pm 1300.43 & \pm 1005.43 & \pm 1022.78 & 3630.57 \\
\hline Enterprise & 1948.01 & 11608.75 & 1801.85 & 1738.35 & 8260.60 & 1622.63 & 2332.65 & 2314.03 & 279.54 & \\
Rd & \pm 302.93 & \pm 751.05 & \pm 802.24 & \pm 776.90 & \pm 2364.73 & \pm 911.44 & \pm 1006.51 & \pm 2357.68 & \pm 417.19 & 3545.16 \\
\hline & 2515.13 & 10656.23 & 4760.27 & 1687.32 & 9468.07 & 1900.57 & 3324.55 & 2420.17 & 449.71 & \\
Bypass & \pm 877.87 & \pm 2366.47 & \pm 5057.4 & \pm 658.97 & \pm 2975.65 & \pm 1068.3 & \pm 1043.42 & \pm 1094.67 & \pm 648.41 & 4131.34 \\
\hline & 2475.47 & 13686.91 & 2635.08 & 1884.55 & 9380.81 & 2107.49 & 3224.94 & 3665.49 & 543.63 & \\
Njiru & \pm 853.71 & \pm 7189.32 & \pm 1018.5 & \pm 607.69 & \pm 1777.97 & \pm 556.08 & \pm 1620.95 & \pm 810.87 & \pm 743.27 & 4400.49 \\
\hline & 2309.05 & 9248.14 & 2534.60 & 1726.40 & 9651.81 & 2089.14 & 2635.29 & 2780.32 & 654.76 & \\
Ruai & \pm 571.07 & \pm 1676.06 & \pm 844.41 & \pm 642.91 & \pm 1066.34 & \pm 620.94 & \pm 909.43 & \pm 810.20 & \pm 874.54 & 3736.61 \\
\hline
\end{tabular}

Figure 2 presents the seasonal variation in the levels o f PBDE congeners in sediment samples from Nairobi River. The concentrations $(\mathrm{ng} / \mathrm{kg}$ ) of these congeners $\mathrm{f}$ or the cooler dry, short rain, hot dry, heavy rain seaso ns are as follows: BDE 28 (16775.18, 21561.29, 10601. 35 and 13287.66); BDE 47 (91149.51, 79440.51, 67841. 373 and 55271.90); BDE 66 (19954.93, 9516.69, 10220. 91 and 16044.32); BDE 85 (7924.16, 11323.09, 11066. 72 and 14134.71); BDE 99 (77271.25, 62410.76, 61811.
29 and 41573.61); BDE 100 (10645.37, 16509.36, 1191 1.14 and 13677.12); BDE 153 (30474.33, 15067.16, 11 475.13 and 15042.5); BDE 154 (31787.23, 14453.72, 13 478.16 and 17904.42); BDE 183 (15038.36, 9963.61, 13 818.61 and 16754.80). The sums of nine PBDE congen ers in sediment were higher during the cooler dry se ason just after the heavy rain this can be attributed to seasonal differential influx of industrial wastes into th e river due to the surfance runoff during the heavy rai n season.

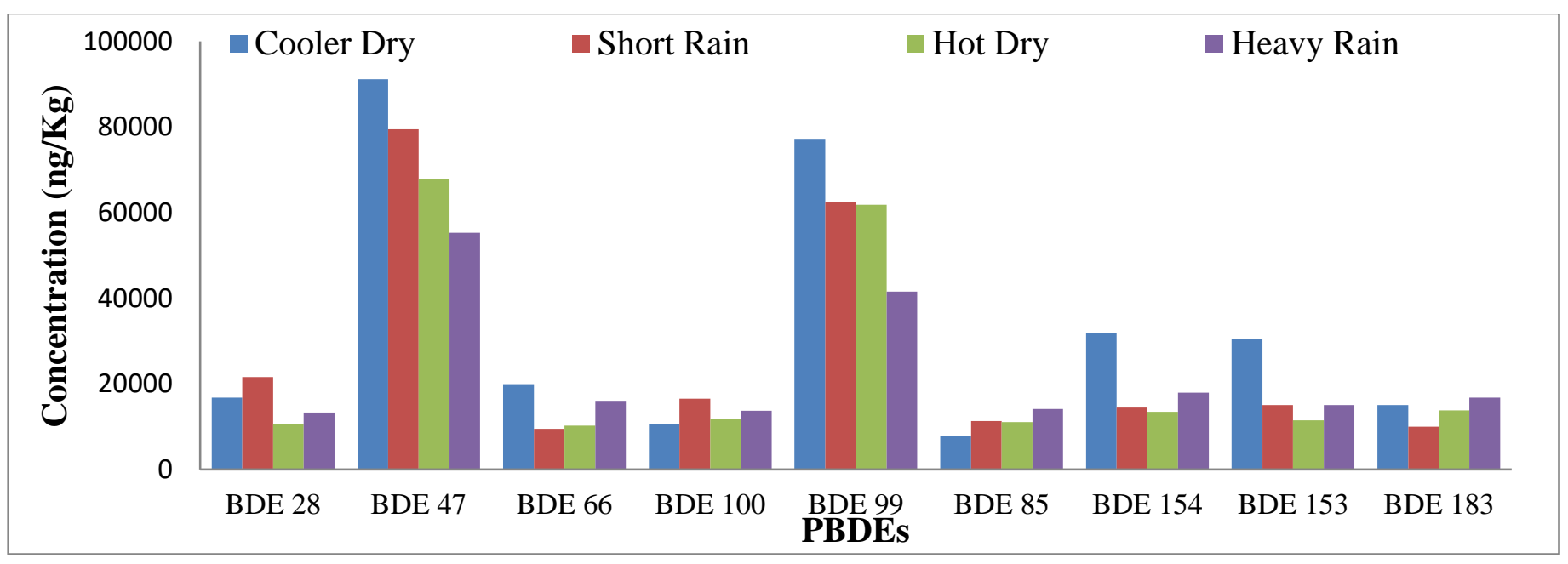

Figure 2 : Seasonal Variation of PBDEs Residue Levels 
PBDEs in sediment samples from Nairobi River basin are likely to come from a combination of point and non-point sources and atmospheric deposition. Larger amounts of industrial activities (like dismantling electronic products, cables and wires, oils depot and chemical plants) were probable accountable for the high levels of PBDEs in sediment samples of Nairobi River. Higher detection frequencies of $100 \%$ for all the PBDE congeners in sediment in this study suggest a widespread distribution of the contaminants in Nairobi River basin. The high levels of penta-BDE formulation in the river sediment can be attributed to inappropriate dumping of polyurethane foam products. The octa-PBDE formulation is mostly used in plastics which when burned release PBDEs into the environment.

All the nine PBDEs congeners studied were commonly identified because they are the major compounds in the penta-BDE commercial mixture [42]. The congener BDE 47 is most frequently employed in the furniture and fabrics [43]. The pentaBDE mixture, which includes BDE 47, 99, 100, 153 and 154 , is typically applied in furniture, whereas the octa and deca-BDEs mixtures are used in the manufacture of many types of polymers, especially those used in televisions, computers, and cables [43].
BDE- 47 accounted for more than $50 \%$ of the total concentration of PBDEs, which showed that it was the most prevalent congener in the sediment samples, followed by BDE 99. According to literature, BDE 47 and 99 are the PBDE congeners that are most frequently detected in environmental samples and biological fluids [44]

\subsection{Comparison of PBDE Residue Levels in the Study}

\section{Area with Levels in Other Countries}

In order to better analyse the results obtained in the current study, the PBDEs levels obtained was compared with the concentrations reported worldwide, as portrayed in Table 4. Compared to some regions in the Fuhe River, the Baiyangdian Lake, and the Chaohu Lake, all of them in China, and on the Olkhon Island, in Russia, the mean concentrations of PBDEs detected in this study were higher. Cheng-Yu, an economic region in China, presented much lower mean values of PBDEs as compared to the Nairobi River investigated herein. Compared to some regions in Korea (four major rivers) or even in China (Jiaojian River), our values were much lower, which means that the Nairobi River sediments were moderately contaminated with PBDEs. A comparison of the concentration of PBDEs in the current study with similar studies around the world is summarized in Table 4.

Table 4 : PBDE Residue Level in Sediment Samples in Other Studies around the World

\begin{tabular}{|c|c|c|c|}
\hline Country & Aquatic System & ¿PBDEs (ng/g) & Reference \\
\hline Brazil & Saibro Lagoon & nd -5.4 & [35] \\
\hline Brazil & Paranoá Lake & $2.5-8.1$ & [45] \\
\hline Chile & Copncepción Bay & $0.02-21$ & [46] \\
\hline China & Fuhe River & $0.13-6.39$ & [47] \\
\hline China & Shanghai rivers & $0.44-12.0$ & [48] \\
\hline China & Jiaojiang River & $8.93-45$ & [36] \\
\hline South Korea & Shihwa Lake & $1.13-18700$ & [37] \\
\hline Canada & Nigara River & $1.10-148$ & [49] \\
\hline USA & White Lake & $0.39-2,4$ & [50] \\
\hline USA & Muskegon Lake & $0.98-3.9$ & {$[50]$} \\
\hline Italy & Maggiore Lake & $0.02-27.1$ & [51] \\
\hline Russia & Olkhon Island & $0.164-0.670$ & [52] \\
\hline Kenya & Nairobi River & $0.134-24.386$ & This study \\
\hline
\end{tabular}


Nd, Not Detected

\section{CONCLUSION}

A lot of works on persistent organic pollutants have been done internationally but there is a scarcity of data about PBDEs in the Kenyan environment. The current research forms a baseline data on the pollution of Nairobi River sediments with PBDEs. The concentration of PBDE congeners increased down the river profile, giving the impression that cumulative human activities play a major role in the contamination of the river. The detection frequency of PBDEs was high during the cool dry season than in the hot dry, heavy rain and short rain seasons respectively. The PBDE levels recorded in this study are of urgent concern because of the potential health impact on the riparian community using the water and river biodiversity.

\section{Disclosure statement}

The authors declare no conflict of interest.

\section{Acknowledgments}

This study was funded by the International Foundation for Science (IFS) grant Number I-2-W6107-1. The authors thank the Research Centre for Toxic Compounds in the Environment (RECETOX), Czech Republic for the donation of the PBDEs standard mix. We also thank the International Science Programme (ISP) whose collaboration lead to the donation of the GC/MS that was used for the analysis of our samples at the University of Nairobi.

\section{REFERENCES}

[1]. Stubbings, W.A., Harrad, S. (2014) Extent and mechanisms of brominated flame retardant emissions from waste soft furnishings and fabrics: a critical review. Environ Int. 71:164175.
[2]. Shaw, S.D., Blum, A., Weber, R., Kannan, K., Rich, D., Lucas, D., Koshland, C.P., Dobraca, D., Hanson, S., Birnbaum, L.S. (2010) Halogenated flame retardants: do the fire safety benefits justify the risks? Rev. Environ. Health 25(4):261-305.

[3]. Besis, A., Samara, C. (2012) Polybrominateddiphenyl ethers (PBDEs) in the indoor and outdoor environments - a review on occurrence and human exposure. Environ. Pollut.169:217-229.

[4]. Gorini, F., Iervasi, G., Coi, A., Pitto, L. and Bianchi, F. (2018) The Role of Polybrominated Diphenyl Ethers in Thyroid Carcinogenesis: Is It a Weak Hypothesis or a Hidden Reality? From Facts to New Perspectives. Int. J. Environ. Res. Public Health 15, 1834.

[5]. Jonathan, C., Kim, G.H., Asa, B., Myriam, G., Andreas, S., Brenda, E. (2010) Polybrominated Diphenyl Ether (PBDE) Flame Retardants and Thyroid Hormone during Pregnancy. Environmental Health Perspectives, 118: 10.

[6]. Khalil A., Cevik S.E., Hung S., Kolla S., Roy M.A. and Suvorov A. (2018) Developmental Exposure to 2,2',4,4'-Tetrabromodiphenyl Ether Permanently Alters Blood-Liver Balance of Lipids in Male Mice. Front. Endocrinol. 9:548.

[7]. Dunnick J.K, Pandiri A.R, Merrick B.A, Kissling G.E, Cunny H, Mutlu E, Waidyanatha S, Sills R, Hong H.L, Ton T.V, Maynor T and Recio L (2018). Carcinogenic activity of pentabrominated diphenyl ether mixture (DE71) in rats and mice. Toxicol rep. 5. 615-624.

[8]. Lee, E., Kim, T.H., Choi, J.S., Nabanata, P., Kim, N.Y., Ahn, M.Y., Jung K.K., Kang, I.H., Kim, T.S., Kwack, S.J., Park, k.L., Kim, S.H., Kang, T.S., Lee, J., Lee, B.M., Kim, H.S. (2010) Evaluation of liver and thyroid toxicity in Sprague-Dawley rats after exposure to polybrominated diphenyl ether BDE-209. J. Toxicol. Sci., 35 (4): 535-545. 
[9]. Law, R.J., Covaci, A., Harrad, S., Herzke, D., Abdallah, M.A., Fernie, K., Toms, L.M., Takigami, H. (2014) Levels and trends of PBDEs and HBCDs in the global environment: status at the end of 2012. Environ Int. 65:147-58.

[10]. Barón, B., Giménez, J., Verborgh, P., Gauffier, P., de Stephanis, R., Eljarrat, E., Barceló, D. (2015) Bioaccumulation and biomagnification of classical flame retardants related halogenated natural compounds and alternative flame retardants in three delphinids from Southern European waters. Environ. Pollut., 203, pp. 107115.

[11]. Andersson, O.B., Blomqvist, G. (1981). Polybrominated aromatic pollutants found in fish in Sweden. Chemosphere 10, 1051-1060.

[12]. Hongwei, S., Yueling, Q Di, Z., Qing, X., Li, D., Jun, W. (2016) Concentrations, distribution, sources and risk assessment of organohalogenated contaminants in soils from Kenya, Eastern Africa. Environmental Pollution 209, 177-185.

[13]. Yao-wen, Q., Dong-Xiao, W., Gan, Z. (2020) Assessment of persistent organic pollutants (POPs) in sediments of the Eastern Indian Ocean. Science of The Total Environment volume 710, 136335.

[14]. Adewuyi, G.O., Adeleye, A.O. (2013) Evaluation of polybrominated diphenyl ethers in sediment of lagos Lagoon, Nigeria. Afr. Environ. Sci. Technol. 7, 686-693.

[15]. Polder, A, Müller, M.B., Lyche, J.L., Mdegela, R. H., Nonga, H.E., Mabiki, F.P., Mbise, T.J., Skaare, J.U., Sandvik, M., Skjerve, E., Lie, E. (2014) Levels and patterns of persistent organic pollutants (POPs) in tilapia (Oreochromis sp.) from four different lakes in Tanzania: geographical differences and implications for human health. Sci. Total Environ., 488-489, pp. 252-260.

[16]. Chokwe, T.B., Okonkwo, J.O., Sibali, L.L., Ncube, E.J., (2015) Alkylphenol ethoxylates and brominated flame retardants in water, fish (carp) and sediment samples from the Vaal River, South Africa. Environ. Sci. Pollut. Res. Int. 22(15):11922-9.

[17]. Olutona, O.G., Oyekunle, O.A.J., Ogunfowokan, O.A., Fatokin, S.O. (2017) Concentrations of polybrominated diphenyl ethers in Water from Asunle Stream, Ile-Ife, Nigeria. Toxics 5: 13.

[18]. Daso, A.P., Fatoki, O.S., Odendaal, J.P. (2013) Occurrence of polybrominateddiphenyl ethers (PBDEs) and 2,2',4,4',5,5'-hexabromobiphenyl (BB-153) in water samples from the Diep River, Cape Town, South Africa. Environ. Sci. Pollut. Res.20: 5168-5176.

[19]. Weiyue, Q., Xinhui, B., Guoying, S., Shaoyou, L., Jiamo, F., Jing, Y., Liping, L. (2007) Exposure to polybrominateddiphenyl ether among workers in electronic waste dismantling region in Guangdong, China. Environment international, 33: 1029-1034.

[20]. Wu, J.P., Luo, X.J., Zhang, Y., Luo,Y., Chen, S.J., Mai, B. X., Yang Z. Y. (2008). Bioaccumulation of polybrominated diphenyl ethers and polychlorinated biphenyls in wild aquatic species from an electronic waste (ewaste) recycling site in South China. Environ Int. 34(8):1109-1113.

[21]. How-Ran, C., Shu-Li, W., Wen-jhy, L., Ya-fen, W., Olaf, P. (2007) Levels of polybrominated diphenyl ether (PBDEs) in breast milk from central Taiwan and their relation to infant birth outcome and maternal menstruation effects. Environment international, 33: 239-245.

[22]. UNEP. (2015) Listing of POPs in the Stockholm Convention Retardants, the Swedish Chemical Society, Stockholm, Sweden.

[23]. Li, W.L., Ma, W.L., Jia, H.L., Hong, W.J., Moon, H.B., Nakata, H., Minh, N.H., Sinha, R.K., Chi, K.H., Kannan, K., Sverko, E., Li, Y.F. (2016) Polybrominated diphenyl ethers (PBDEs) in surface soils across five Asian countries: levels, 
spatial distribution, and source contribution. Environ. Sci. Technol. 50, 12779-12788.

[24]. Ndiritu, G. G., Gichuki, N. N., Kaur, P. and Triest, L. (2003) Characterization of environmental gradients using physicochemical measurements and diatom densities in Nairobi River, Kenya. Aquatic Ecosystem Health \& Management, 6 (3): 343-354.

[25]. UNEP. (2009) "Kenya: Atlas of Our Changing Environment." Division of Early Warning and Assessment (DEWA), United Nations Environment Programme (UNEP). Nairobi.

[26]. Nduda, E.N., Madadi, V.O., Wandiga S.O. (2018) Organochlorine pesticide residues in sediment and water from Nairobi River, Kenya: levels, distribution, and ecological risk assessment. Environ Sci Pollut Res Int. 25(34): 34510-34518.

[27]. Masese, F. (2010) Investigation of pollutants, determination of physiochemical characteristics of Nairobi River and remediation of some toxic heavy metals using fish bone. Master's thesis, University of Nairobi, Nairobi, Kenya.

[28]. Budambula, N.L., Mwachiro, E.C. (2005) Metal Status of Nairobi River Waters and their Bioaccumulation in Labeo Cylindricus. Water, Air, and Soil Pollution.169: 275-291.

[29]. Makokha VA, Ndung'u AW, Mungai TM, Yan X, Wang J (2018) Concentrations, Sources, and Risk Assessment of Organohalogen Compounds in Soils from Kiambu to Mombasa, Kenya. Bull Environ Contam Toxicol 101, 766-772.

[30]. Kenya Meteorological Department. (2018) The outlook for the October-November-December 2018 season and review of rainfall during the "long rains" (March to May) and June-JulyAugust 2018 seasons. Government press.

[31]. UNEP (2010). UNEP chemicals POPs analysis training project. Four day practical training course in Amsterdam at the institute for environmental studies. VU University. March 8-13. 2010.
[32]. Hiller E, Zemanová L, Sirotiak M, Jurkovič L (2011). Concentrations, distributions, and sources of polychlorinated biphenyls and polycyclic aromatic hydrocarbons in bed sediments of the water reservoirs in Slovakia. Environ Monit. Assess, 173:883-897.

[33]. Floehr T, Xiao H, Scholz-Starke B, Wu L, Hou J, Yin D, Zhang X, Ji R, Yuan X, Ottermanns R, Roß-Nickoll N, Schäffer A, Hollert H (2013). Solution by dilution? A review on the pollution status of the Yangtze River. Environ. Sci. Pollut. Res. 20:6934-6971.

[34]. Olutona G.O, Oyekunle J.A.O, Ogunfowokan A.O and Fatoki O.S (2016). Assessment of polybrominated diphenyl ethers in sediment of Asunle Stream of the Obafemi Awolowo University, Ile-Ife, Nigeria. Environ. Sci. Pollut. Res., 23: 21195-21205.

[35]. Ferrari R.S, de Souza A.O, Annunciação D.L.R, Sodré F.F and Dorta J.D (2019). Assessing Surface Sediment Contamination by PBDE in a Recharge Point of Guarani Aquifer in Ribeirão Preto, Brazil. Water, 11: 1601.

[36]. Yang S, Fu Q Teng M, Yang J (2015). Polybrominated diphenyl ethers (PBDEs) in sediment and fish tissues from Lake Chaohu, central eastern China. Arch. Environ. Prot., 41:12.

[37]. Moon H.B, Choi M, Yu J, Jung R.H and Choi H.G (2012). Contamination and potential sources of polybrominated diphenyl ethers (PBDEs) in water and sediment from the artificial Lake Shihwa, Korea. Chemosphere, 88: 837

[38]. Lacorte S, Guillamón M, Martínez E, Viana, P, Barceló D (2003). Occurrence and specific congener profile of 40 polybrominated diphenyl ethers in river and coastal sediment from Portugal. Environ. Sci. Technol 37:892-898.

[39]. Ohta S, Nakao T, Nishimura H, Okumura T, Aozasa O, Miyata H (2002). Contamination levels of PBDEs, TBBPA, PCDDs/DFs, 
PBDDs/DFs, and PXDDs/Fs in the environment of Japan. Organohalogen Compounds, 57:57-60.

[40]. Oros D.R, Hoover D, Rodigari F, Crane D, Sericano J (2005). Levels and distribution of polybrominated diphenyl ethers in water, surface sediments, and bivalves from the San Francisco Estuary. Environ. Sci. Technol. 537(39):33-41.

[41]. Environment Canada (2013). Canadian environmental protection act, 1999, Federal environmental quality guidelines polybrominated diphenyl ethers (PBDEs). Environment Canada, Gatineau.

[42]. Streets S.S, Henderson S.., Stoner A.D, Carlson D.L, Simcik M.F, Swackhamer D.L (2006). Partitioning and bioaccumulation of PBDEs and PCBs in Lake Michigan. Environ. Sci. Technol, 40: 7263-7269.

[43]. Darnerud PO (2003). Toxic effects of brominated flame retardants in man and in wildlife. Environ. Int., 29(6):841-53.

[44]. Botaro D, Torres J.P.M (2007). Difenil éteres polibromados (PBDEs) - Novos poluentes, antigos desafios. Oecologia Bras, 11:167.

[45]. Reistad T, Mariussen E.A (2005). Commercial mixture of the brominated flame retardant pentabrominated diphenyl ether (de-71) induces respiratory burst in human neutrophil granulocytes in vitro. Toxicol Sci. 87(1):57-65.

[46]. Annunciação D.L.R., Almeida F.V, Sodré F.F (2017). Method development and validation for the determination of polybrominated diphenyl ether congeners in Brazilian aquatic sediments. Microchem. J. 133: 43.

[47]. Pozo K, Kuku `cka $\mathrm{P}, \mathrm{Va}$ `nková L, P`ribylová P, Klánová J, Rudolph A, Banguera Y, Monsalves J, Contreras S, Barra R (2015). Polybrominated Diphenyl Ethers (PBDEs) in Concepción Bay, central Chile after the 2010 Tsunami. Mar. Pollut. Bull, 95: 480.

[48]. Hu G, Xu Z, Dai J, Mai B, Cao H, Wang J, Shi Z, Xu M (2010). Distribution of polybrominated diphenyl ethers and decabromodiphenylethane in surface sediments from Fuhe River and Baiyangdian Lake, North China. J. Environ. Sci, 22: 1833.

[49]. Wang X.-T, Chen L, Wang X.-K, Zhang Y, Zhou J, Xu S.-Y, Sun Y.-F, Wu M.-H (2015). Occurrence, profiles, and ecological risks of polybrominated diphenyl ethers (PBDEs) in river sediments of Shanghai, China. Chemosphere, 133: 22

[50]. Samara F, Tsai C.W, Aga D.S (2006). Determination of potential sources of PCBs and PBDEs in sediments of the Niagara River. Environ. Pollut. 139: 489.

[51]. Bradley P.W, Wan Y, Jones P.D, Wiseman S, Chang H, Lam M.H.W, Long D.T, Giesy J.P (2011). PBDEs and methoxylated analogues in sediment cores from two Michigan, USA, inland lakes. Environ. Toxicol. Chem. 30: 1236.

[52]. Mariani G, Canuti E, Castro-Jiménez J, Christoph E.H, Eisenreich S.J, Hanke G, Skejo H, Umlauf G (2008). Atmospheric input of POPs into Lake Maggiore (Northern Italy): PBDE concentrations and profile in air, precipitation, settling material and sediments. Chemosphere, 73:114.

[53]. Ok G, Shirapova G, Matafonova G, Batoev V, hyung Lee S (2013). Characteristics of PAHs, PCDD/Fs, PCBs and PBDEs in the sediment of Lake Baikal, Russia. Polycycl. Aromat. Compd. 33: 173.

Cite this article as : Enock M. Osoro, Shem O. Wandiga, Vincent O. Madadi, Deborah A. Abong'o, "Occurrence and Distribution of Polybrominated Diphenyl Ethers in Sediments from Nairobi River Basin, Kenya, East Africa", International Journal of Scientific Research in Science, Engineering and Technology (IJSRSET), Online ISSN : 2394-4099, Print ISSN : 2395-1990, Volume 8 Issue 1, pp. 274-286, January-February 2021. Available at doi : https://doi.org/10.32628/IJSRSET218148 Journal URL : https://ijsrset.com/IJSRSET218148 